\title{
INVESTING AND STOPPING
}

\author{
MORITZ DUEMBGEN *** AND \\ L. C. G. ROGERS, ${ }^{*}$ University of Cambridge
}

\begin{abstract}
In this paper we solve the hedge fund manager's optimization problem in a model that allows for investors to enter and leave the fund over time depending on its performance. The manager's payoff at the end of the year will then depend not just on the terminal value of the fund level, but also on the lowest and the highest value reached over that time. We establish equivalence to an optimal stopping problem for Brownian motion; by approximating this problem with the corresponding optimal stopping problem for a random walk we are led to a simple and efficient numerical scheme to find the solution, which we then illustrate with some examples.
\end{abstract}

Keywords: Optimal investment; optimal stopping; Brownian motion

2010 Mathematics Subject Classification: Primary 60G40

Secondary $62 \mathrm{~L} 15$

\section{Introduction}

The fee structure of a hedge fund typically consists of two components, a fixed management fee (this will usually be a relatively low percentage, $2 \%$ being common), charged on all assets under management, and a performance fee (this is usually charged at quite a high rate, $20 \%$ being common), charged on any gain achieved on the funds invested. The exact contractual agreement has to specify between what dates the gain must have been recorded, what happens to the management fee for funds that are deposited for only part of the full period of reckoning, and many other details, such as any restrictions on investors' freedom to withdraw funds with or without notice periods. We shall simplify the problem here by assuming that the performance fees are charged at the end of each year on all funds held at the end of the year. (It may be that some funds are withdrawn before the end of the year, and could in principle be liable to pay performance fees, but we shall ignore this, on the grounds that investors would be unlikely to withdraw funds while they were ahead.) The gain is calculated as the increase in value of the funds from the time they were deposited in the fund, or from the beginning of the year, whichever is later. Thus, the baseline for calculating the performance fee resets at the beginning of each year. We shall suppose that the management fee is charged only on the funds still under management at the end of the year; this is a simplification, but as the management fee is typically of smaller magnitude, it is relatively innocent.

In principle, the total fees charged at the end of the year by the hedge fund to its clients would depend on the entire history of investments and withdrawals throughout the year, as well as on the actual performance path of the hedge fund. We shall propose a simplified mechanism for this, which involves modelling how the quantity of assets under management (AUM) varies

Received 22 October 2012; revision received 10 November 2013.

* Postal address: Statistical Laboratory, University of Cambridge, Cambridge CB3 0WB, UK. Email address:

1.c.g.rogers@statslab.cam.ac.uk

** Email address: m.duembgen@gmail.com 
as the level of the hedge fund fluctuates, and is explained in detail in Section 2. This model captures the key features that the AUM rises as the level of the fund rises, and falls as the level falls; that newly invested funds enter at the current level; and that funds withdrawn will have entered the fund at a level above the current level. The model we present is not perfect, but it has the crucial simplifying property that the fees paid will depend on the level of the fund at the start of the year, at the end of the year, and on the highest and lowest levels attained. This saves us from needing to carry along as a state variable the entire profile of the levels at which the current AUM entered the fund, which would then be impossibly clumsy to work with; cf. the work of Dybvig and Koo [3] on wash sales).

In a seminal contribution to this subject Goetzmann et al. [4] provided closed-form solutions to a model which differs from ours, namely that the performance fee is considered to be paid out continuously over time (with a high-water mark provision). This has the undesirable side effect that, at the end of the year, the manager's reward is a function only of the high-water mark. Guasoni and Obloj [5] followed a similar approach (with a continuously paid performance fee), but modelled the manager as a utility optimiser; this also resulted in (asymptotic) closed-form solutions.

Accepting this simplified model, we find ourselves with an optimal control problem for the hedge fund manager, in which the objective is a function of the initial, final, highest, and lowest values taken by the controlled process throughout the year. We shall suppose that the riskless rate is zero and that the hedge fund manager is, in fact, just investing in assets which fluctuate but have no drift. This ignores a fund manager's presumed ability to pick winners, time the market, anticipate roll dates, or any other marketing boast; this may be unjust at the level of a single talented manager, but not too far from the situation for the industry as a whole. An alternative justification is that while the invested assets might have a positive drift, the manager, as a risk control measure, will take expectations under an equivalent measure which removes the drift.

In our model the level of the fund will therefore evolve as a martingale, which, for simplicity, we suppose is continuous; the manager can adjust the volatility of the level process by changing the position of the risky assets, but he cannot affect the drift. Nevertheless, he has an incentive to embrace some risk, as he has a call option interest in the level of the fund, as well as the performance fee incentive. We shall begin our analysis by converting the manager's problem into an optimal stopping problem for a Brownian motion, since any continuous martingale is a time change of Brownian motion. This step is not quite as obvious as it seems at first sight, as we explain in Section 2.1. The next step is to convert the optimal stopping problem for Brownian motion into an optimal stopping problem for a symmetric simple random walk (SSRW), whose value will be close to the value of the original problem; the difference is analyzed and estimated in Section 2.2. While it would be possible to write down some formulation of the solution to the original continuous problem, it would not be particularly digestible, and there would then be the issue of existence and uniqueness of solutions. Since we do not ever expect to be able to exhibit any closed-form solution, we are forced to employ numerical methods to gain an understanding, and these are naturally discrete in nature. Our estimates allow us to be quite precise about the error incurred by the approximation. In Section 2.3 we solve this problem numerically.

In Section 3 we return to the hedge fund manager's problem, where we state our modelling assumptions on how cash flows into and out of the fund as the level of the fund varies, converting the manager's objective into one of the type studied in Section 2. In Section 3 we present numerical solutions of this problem and in Section 4 we present our conclusions. 


\section{From investing to stopping}

In this section we show that the investment problem can be recast as a stopping problem for Brownian motion. We show that this stopping problem can be approximated by the corresponding stopping problem for an SSRW and we explain the algorithm for solving this SSRW.

\subsection{The investing/stopping equivalence}

We suppose that the level of the fund is $w_{0}$ at time 0 , and evolves as

$$
\mathrm{d} w_{t}=\theta_{t} \mathrm{~d} W_{t}
$$

for some previsible process $\theta$ for which the stochastic integral is defined, where $W$ is a standard Brownian motion. We define

$$
\underline{w}_{t} \equiv \inf \left\{w_{s}: s \leq t\right\}, \quad \bar{w}_{t} \equiv \sup \left\{w_{s}: s \leq t\right\},
$$

and we suppose that the objective of the manager of the fund is

$$
\sup _{\theta} \mathbb{E} F\left(\underline{w}_{1}, w_{1}, \bar{w}_{1}\right)
$$

for an $F:\left(-\infty, w_{0}\right] \times \mathbb{R} \times\left[w_{0}, \infty\right) \rightarrow \mathbb{R}$ for which the above expectation is always well defined (continuous and bounded from above or below, say). The well-known Dubins-Schwarz result says (informally) that any continuous local martingale is a time change of a Brownian motion. More precisely, if we extend the definition (2.1) of $w$ beyond time 1 by setting $\theta_{t}=1$ for all $t \geq 1$, and set

$$
A_{t} \equiv \int_{0}^{t} \theta_{s}^{2} \mathrm{~d} s, \quad \tau_{t} \equiv \inf \left\{s: A_{s}>t\right\},
$$

then $B_{t} \equiv w\left(\tau_{t}\right)$ defines a Brownian motion relative to the filtration $g_{t} \equiv \mathcal{F}_{\tau_{t}}$, and each $A_{t}$ is a $g$-stopping time. It follows directly that

$$
w_{t}=B\left(A_{t}\right), \quad \underline{w}_{t}=\inf _{0 \leq s \leq A_{t}} B_{s} \equiv \underline{B}\left(A_{t}\right), \quad \bar{w}_{t}=\sup _{0 \leq s \leq A_{t}} B_{s} \equiv \bar{B}\left(A_{t}\right) .
$$

As a consequence, were it not for the fact that $A_{1}$ is not, in general, a stopping time for $B$, the following result would be trivial.

Lemma 2.1. For a continuous $F$, bounded from above or below, the equality

$$
\sup _{\theta} \mathbb{E} F\left(\underline{w}_{1}, w_{1}, \bar{w}_{1}\right)=\sup _{T \in \mathcal{T}} \mathbb{E} F\left(\underline{B}_{T}, B_{T}, \bar{B}_{T}\right)
$$

is valid, where $\mathcal{T}$ denotes the set of stopping times of the Brownian motion $B$.

Proof. See Appendix A.

Remark 2.1. Suppose that $M$ is a continuous martingale which runs like a Brownian motion until some independent exponential random time $T$, then stands still for one unit of time, and then resumes Brownian motion. The quadratic variation process $[M]$ grows at rate 1 except in the interval $[T, T+1]$, where it remains constant. It is quite easy to show that $[M]_{T}$ is a $g$-stopping time, but it is impossible to discover what $[M]_{T}=T$ is just by looking at the time-changed Brownian path $B_{t}=M\left(\tau_{t}\right)$. Thus, we expect the left-hand side of (2.3) to be at least as large as the right-hand side, but it is not initially obvious that the two sides are the same. 


\subsection{Approximation by random walk}

Thanks to Lemma 2.1, we are now left to solve an optimal stopping problem for a Brownian motion whose stopping reward is a function of its current value, minimum, and maximum

$$
V=\sup _{T \in \mathcal{T}} \mathbb{E} F\left(\underline{B}_{T}, B_{T}, \bar{B}_{T}\right) .
$$

Although suppressed in the notation, we think of $V$ as a function of the starting values $X_{0} \equiv\left(\underline{B}_{0}, B_{0}, \bar{B}_{0}\right)$. It should come as no surprise that we can approximate $V$ uniformly by a stopping problem for an SSRW $w^{h}$ on the grid $B_{0}+h \mathbb{Z}$

$$
V^{h}=\sup _{T \in \hat{\mathcal{T}}} \mathbb{E} F\left(\underline{w}_{T}^{h}, w_{T}^{h}, \bar{w}_{T}^{h}\right),
$$

where $\hat{\mathcal{T}}$ represents all (discrete) $w^{h}$-stopping times.

Lemma 2.2. Let $F$ be uniformly continuous: there is some continuous function $\psi$ tending to zero at zero such that for all $x, x^{\prime}$

$$
\left|F(x)-F\left(x^{\prime}\right)\right| \leq \psi\left(\left\|x-x^{\prime}\right\|\right) .
$$

If the optimization problem is well posed, then

$$
\left|V^{h}\left(X_{0}\right)-V\left(X_{0}\right)\right| \leq \psi(h \sqrt{3}) .
$$

Proof. See Appendix B.

\subsection{Solving the random walk stopping problem}

Given that we have now replaced the original investment problem with an optimal stopping problem for an SSRW, we are in a position to solve it by numerical means. (It is inconceivable that we may be able to find closed-form solutions, except in some very contrived examples.) If we are to follow this route then we will, of course, only be able to deal with examples which are finite. For this reason we are justified in assuming that the random walk will be stopped once it leaves some interval $\left(w_{*}, w^{*}\right)$ containing $w_{0}$.

Now suppose that $w_{*}=w_{0}-m h, w^{*}=w_{0}+n h$ for some positive integers $m, n$, and introduce the notation

$$
F_{j k}(i)=F\left(w_{0}-j h, w_{0}+i h, w_{0}+k h\right), \quad V_{j k}(i)=V^{h}\left(w_{0}-j h, w_{0}+i h, w_{0}+k h\right)
$$

for $-m \leq-j \leq i \leq k \leq n$. We have that $V_{j k} \geq F_{j k}$ always, and that if $j=m$ or $k=n$ equality holds, since the random walk must have stopped by the time it reaches those points. We can now solve recursively for the value function $V$ rather as we would solve a dynamic programming problem. We obtain, for $-j<i<k$,

$$
V_{j k}(i)=\max \left\{F_{j k}(i), \frac{1}{2}\left(V_{j k}(i+1)+V_{j k}(i-1)\right)\right\}
$$

and at the ends of the interval, we have

$$
\begin{aligned}
V_{j k}(-j) & =\max \left\{F_{j k}(-j), \frac{1}{2}\left(V_{j k}(-j+1)+V_{j+1, k}(-j-1)\right)\right\}, \\
V_{j k}(k) & =\max \left\{F_{j k}(k), \frac{1}{2}\left(V_{j, k+1}(k+1)+V_{j k}(k-1)\right)\right\} .
\end{aligned}
$$




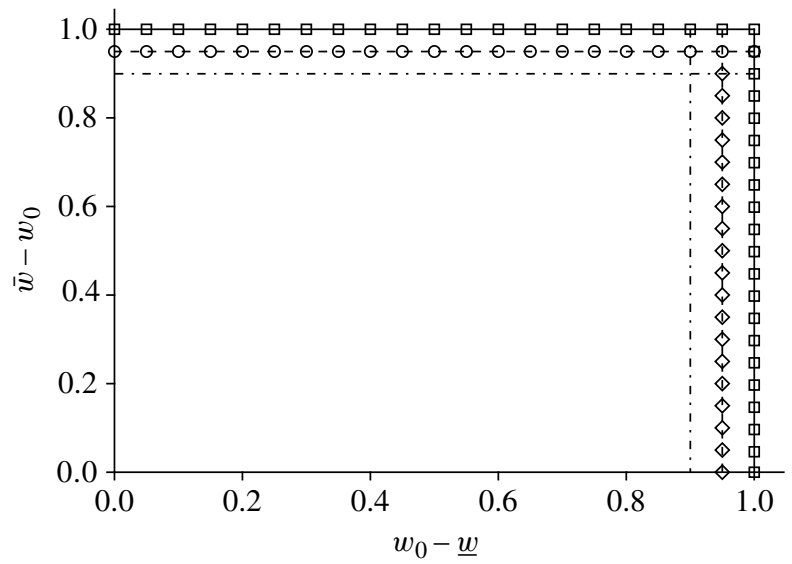

FIGURE 1: Filling in the value function. Here, $w^{*}-w_{0}=w_{0}-w_{*}=1$.

The situation is illustrated in Figure 1, where we plot the grid of $(\underline{w}, \bar{w})$ pairs. We may imagine that we are looking down on a cube, each point of the form $\left(w_{0}-j h, w_{0}+k h\right)$ being the projection down into the plane of points of the form $\left(w_{0}-j h, w_{0}+i h, w_{0}+k h\right),-j \leq i \leq k$. At every point of the upper-right boundary of the rectangle, where either $j=m$ or $k=n$, the value function is equal to $F$ and is therefore known. Now we work out the values $V_{m-1, n-1}(i)$, by solving the optimal stopping problem (2.4) with the boundary conditions (2.5) and (2.6). The two boundary conditions require knowledge of $V$ at $(m-1, n)$ and $(m, n-1)$; but these values are known, since we know $V=F$ on the solid upper-right boundary of the rectangle. Now we calculate the value of $V_{m-2, n-1}$; this time, we need to know $V$ at $(m-2, n)$ (where it agrees with $F)$ and $(m-1, n-1)$ (which we calculated at the first step). Continuing in this fashion, we are able to calculate the values of $V$ at all points of the form $(\ell, n-1)$, represented by circles in Figure 1. Similarly, we can work out the values of $V$ at all points $(m-1, \ell)$ marked with diamonds, which gives us the values of $V$ not only at the upper-right boundary, but at the next layer in, depicted by the circles and diamonds in the diagram. But now we have reduced the size of the rectangle by one in each direction, so we can repeat the method to find all the values of $V$ on the dot-dash lines. Proceeding similarly gives us the solution $V$.

Remark 2.2. At each node $(j, k)$ of the rectangle, shown in Figure 1 , we have to solve an optimal stopping problem for a random walk in $\{i:-j-1 \leq i \leq k+1\}$, where the random walk is absorbed at the endpoints $-j-1$ and $k+1$, with values $V_{j+1, k}(-j-1)$ and $V_{j, k+1}(k+1)$, respectively, and with stopping values $F_{j k}(i)$ at interior points. The value has a geometric interpretation as the least concave majorant of the function defined by the stopping values, and can be calculated rapidly and accurately by policy improvement.

Remark 2.3. It may happen that for a given $(j, k)$ the optimal stopping solution is not to stop in the interior, otherwise there will be a smallest value $w_{0}+q h=\eta_{l}(j, k)$ and a largest value $w_{0}+\ell h=\eta_{u}(j, k)$ at which $V_{j k}(i)=F_{j k}(i)$. By convention, we may define $\eta_{l}(j, k)=$ $w_{0}+(k+1) h$ and $\eta_{u}(j, k)=w_{0}-(j+1) h$ if the optimal stopping solution does not admit stopping in the interior of the interval. At times $\tau$ when the random walk reaches a new maximum $w_{\tau}=\bar{w}_{\tau}=w_{0}+k h$, it will thereafter continue until either it hits $w_{\tau}+h$ or $\eta_{u}(j, k)$, where $\underline{w}_{\tau}=w_{0}-j h$. If it hits the lower barrier $\eta_{u}(j, k)$ before it hits $w_{0}+(k+1) h$, then it will stop there for good, unless $\eta_{u}(j, k)=w_{0}-(j+1) h$, in which case a new minimum has been achieved, and the random walk can continue to move. 


\section{The hedge fund manager's investing problem}

We return to the problem, introduced in Section 2, of the hedge fund manager, who can control the level $w_{t}$ of the fund through the position $\theta_{t}$ in the risky asset. As the level of the fund goes up and down, the assets under management vary. We propose a very simple model for this which allows us to represent the manager's problem in the form (2.2), which can then be solved by the techniques just presented.

The basic idea is that there is some $C^{1}$ nonnegative function $\varphi$ such that any time $\tau$ when the level process $w$ is at its running maximum, $w_{\tau}=\bar{w}_{\tau}$, the profile of the basis levels of the assets in the fund should be given by

$$
\varphi(x) \mathrm{d} x, \quad 0 \leq x \leq \bar{w} .
$$

So, in particular, the total assets under management at $\tau$ would be $\Phi(\bar{w}) \equiv \int_{0}^{\bar{w}} \varphi(x) \mathrm{d} x$. If we demanded that $\varphi$ was increasing, this would represent a situation where the more successful the fund, the more people would bring their money into it.

What happens as the level of the fund falls back from its running maximum? Investors will take their money out of the fund; as the level rises again, investors will put money in. Now as the level rises again and new money comes into the fund the basis at which that new money was invested has to be the current level. In order to retain tractability, we shall insist that when money is withdrawn from the fund, as the level falls, it is removed only at the current level. This is a restrictive assumption, but we make it nevertheless. So as the level falls, a fraction $(1-p)$ of the assets invested at the current level are removed from the fund, so that in general the profile of basis values in the fund is

$$
\varphi(x) \mathbf{1}_{\left\{x \leq w_{t}\right\}}+p \varphi(x) \mathbf{1}_{\left\{w_{t} \leq x \leq \bar{w}_{t}\right\}},
$$

which is consistent with (3.1) when $w_{t}=\bar{w}_{t}$. The assumption we make means that if the level of the fund falls a long way there will still be quite a lot of assets which came in at a higher level and have not yet been taken out. This could be understood in terms of the reluctance of investors to realize a loss; investors would be willing to come out at zero gain, and they do in our model, but they would never come out if they would thereby realize a loss.

This is not the whole model because the performance-related part of the manager's fees will be measured relative to the level $w_{0}$ of the fund at the start of the year. We shall therefore suppose that initially the profile of basis levels is a point mass at $w_{0}$ of magnitude $\Phi\left(w_{0}\right)$, and that if the level falls to $\underline{w}$ then the funds $(1-p)\left(\Phi\left(w_{0}\right)-\Phi(\underline{w})\right)$ which would be removed if the profile $\varphi$ extended through $\left(0, w_{0}\right)$ will be removed from the atom at zero. Thus, when the minimum value of the level is $\underline{w}$, the size of the atom at $w_{0}$ will be

$$
\Phi\left(w_{0}\right)-(1-p)\left(\Phi\left(w_{0}\right)-\Phi(\underline{w})\right)=p \Phi\left(w_{0}\right)+(1-p) \Phi(\underline{w}) .
$$

Thus, overall the profile of the basis levels will be

$$
\left[\varphi(x) \mathbf{1}_{\left\{\underline{w}_{t} \leq x \leq w_{t}\right\}}+p \varphi(x) \mathbf{1}_{\left\{w_{t} \leq x \leq \bar{w}_{t}\right\}}\right] \mathrm{d} x+\left\{p \Phi\left(w_{0}\right)+(1-p) \Phi(\underline{w})\right\} \delta_{w_{0}} .
$$

Integrating this gives the total assets under management as

$$
\begin{aligned}
\mathrm{AUM} & =\Phi(w)-\Phi(\underline{w})+p(\Phi(\bar{w})-\Phi(w))+p \Phi\left(w_{0}\right)+(1-p) \Phi(\underline{w}) \\
& =(1-p) \Phi(w)+p(\Phi(\bar{w})-\Phi(\underline{w}))+p \Phi\left(w_{0}\right) .
\end{aligned}
$$


We shall suppose that there is some constant $\beta \in(0,1)$ such that the manager receives

$$
\mathrm{MF}=\beta \times \mathrm{AUM}
$$

as the management fee. The profile (3.2) allows us to calculate the performance component of the manager's reward, which will be

$$
\mathrm{PF}=\alpha\left[\int_{\underline{w}}^{w}(w-x) \varphi(x) \mathrm{d} x+\left(w-w_{0}\right)^{+}\left\{p \Phi\left(w_{0}\right)+(1-p) \Phi(\underline{w})\right\}\right] .
$$

\subsection{Numerical examples}

We suppose that the manager is risk averse, so he/she tries to maximize

$$
\mathbb{E} U(\mathrm{MF}+\mathrm{PF})
$$

In this first example, we take $U=\log (x), \varphi=\sqrt{x \wedge K}, \alpha=20 \%, \beta=2 \%, p=0.3$, $w_{0}=1, \Phi\left(w_{0}\right)=1$, and $K=3$. Figures 2 and 3 illustrate the resulting payout function $F=U(\mathrm{MF}+\mathrm{PF})$ as a function of the three variables $(\underline{w}, w, \bar{w})$. Since it is decreasing in $\underline{w}$, increasing in $\bar{w}$, as well as S-shaped in $w$ (first convex, then concave), we expect nontrivial results from the stopping problem.

It is worth understanding why this should be. For fixed $(\underline{w}, \bar{w})$, the optimal stopping problem is on the grid $\{\underline{w}-h, \underline{w}, \ldots, \bar{w}, \bar{w}+h\}$, where if we stop at $x \in[\underline{w}, \bar{w}]$, we get reward $F(\underline{w}, x, \bar{w})$, but if we stop at one of the endpoints, we get (at the upper endpoint for example) $F(\underline{w}, \bar{w}+h, \bar{w}+h)$. This value can be (and in places is) significantly larger than $F(\underline{w}, \bar{w}, \bar{w})$, so we see a plot like Figure 4. But if the values at the endpoints are somewhat lower, there can be stopping in the interior.

Figure 4 illustrates the typical situation for values $(\underline{w}, \bar{w})$ close enough to $\left(w_{0}, w_{0}\right)$ that it is beneficial to keep going; the set of such values we call the continuation region. Figure 5 illustrates the situation once $(\underline{w}, \bar{w})$ has moved sufficiently far from $\left(w_{0}, w_{0}\right)$.

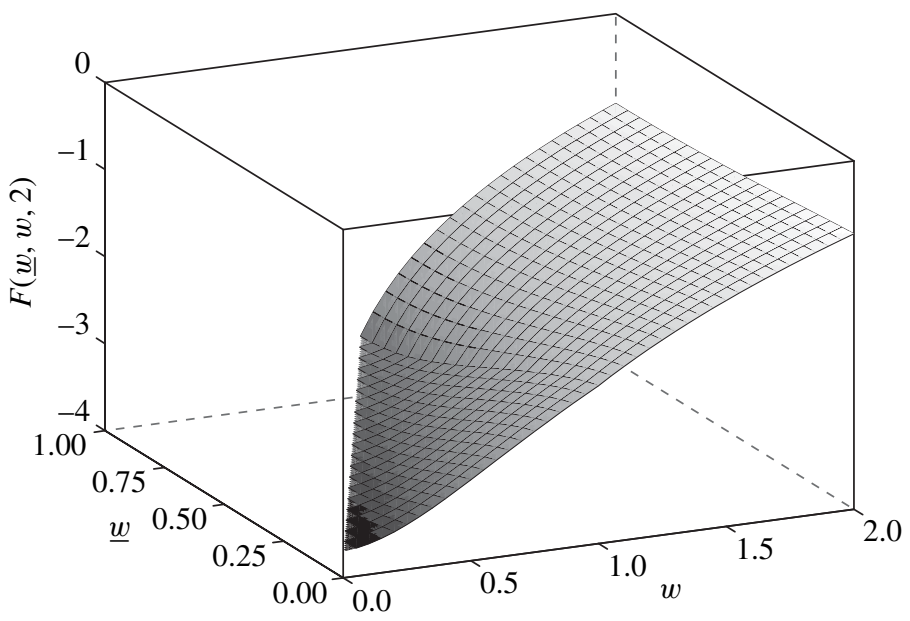

Figure 2: A graph of $F(\underline{w}, w, \bar{w})$ for fixed $\bar{w}=2$ : S-shaped in $w$ and decreasing in $\underline{w}$. 


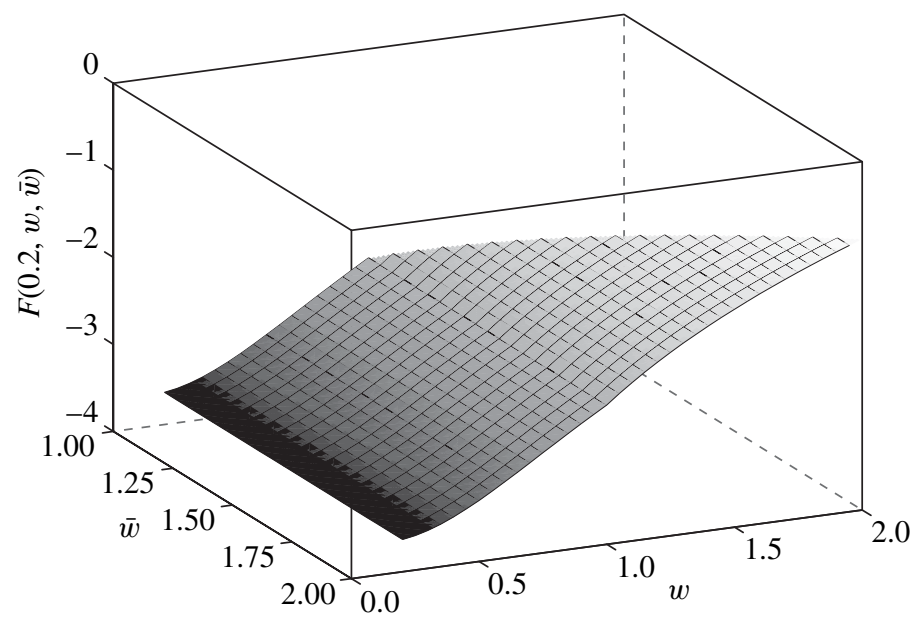

Figure 3: A graph of $F(\underline{w}, w, \bar{w})$ for fixed $\underline{w}=0.2$ : S-shaped in $w$ and slightly increasing in $\bar{w}$.

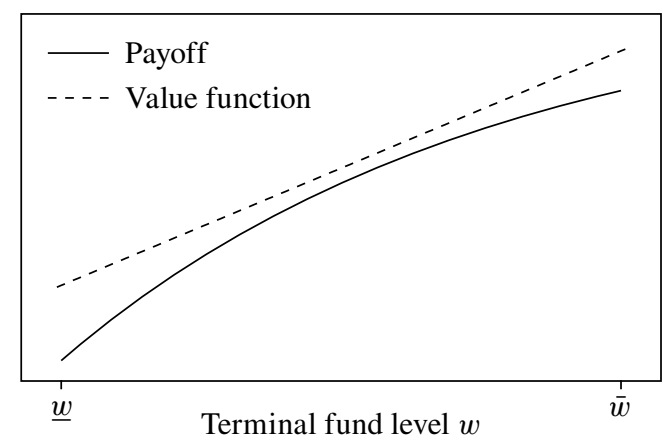

FIGURE 4: Payoff and value function for fixed $(\underline{w}, \bar{w})$ in the continuation region.

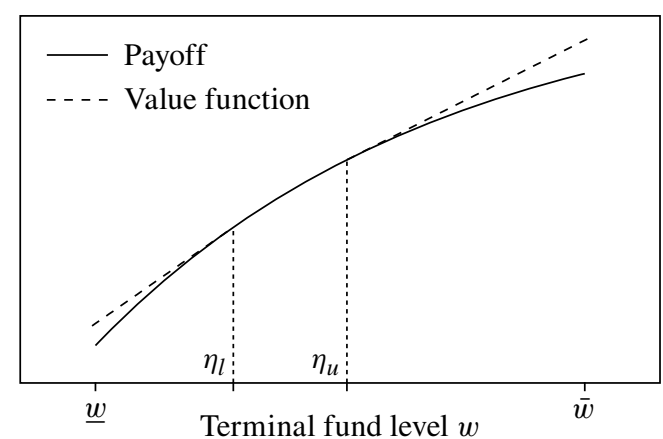

FIGURE 5: Payoff and value function for fixed $(\underline{w}, \bar{w})$ outside the continuation region. 


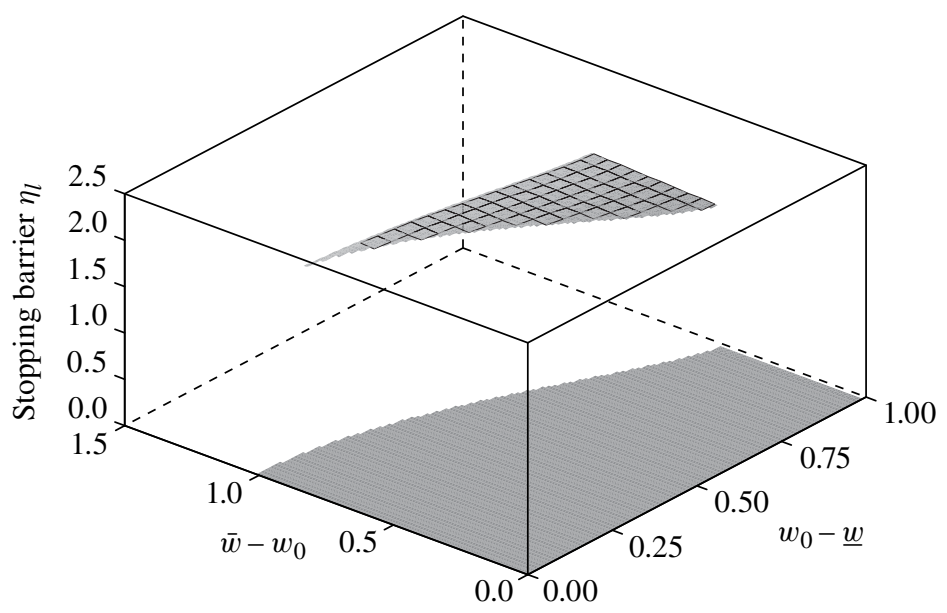

FIGURE 6: Surface plot of the upper stopping barrier $\eta_{l}$ that becomes relevant when we leave the continuation region (grey area) by decreasing $\underline{w}$.

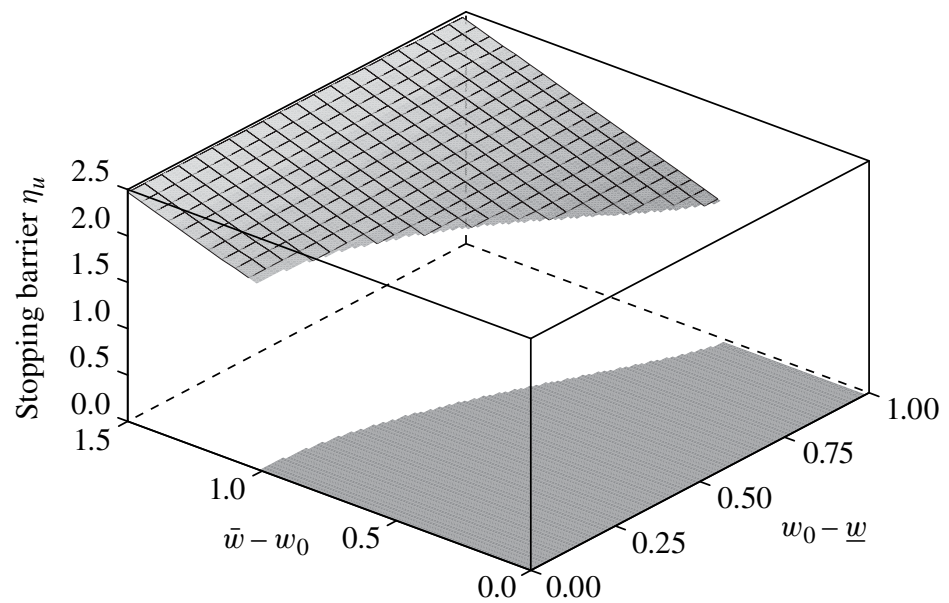

FIGURE 7: Surface plot of the lower stopping barrier $\eta_{u}$ that becomes relevant when we leave the continuation region (grey area) by increasing $\bar{w}$.

Figures 6 and 7 plot out the continuation region and the barriers $\eta_{l}, \eta_{u}$ for our first example. Various comments are in order.

(i) If, for some $(\underline{w}, \bar{w})$, there is optimal stopping at some interior value then both $\eta_{l}<\bar{w}+h$ and $\eta_{u}>\underline{w}-h$. It is not possible to have $\eta_{l}<\bar{w}+h$ and $\eta_{u}=\underline{w}-h$; or $\eta_{u}>\underline{w}-h$ and $\eta_{l}=\bar{w}+h$. Note that if $\eta_{l}<\bar{w}+h$ (and, therefore, $\left.\eta_{u}>\underline{w}-h\right)$, we always have $\eta_{l} \leq \eta_{u}$.

(ii) In the computed plots, the continuation region is a connected set. In general, for an optimal stopping problem with stopping reward $g(\underline{w}, w, \bar{w})$ this does not need to happen.

(iii) In the computed plots, we have the property that if $(\underline{w}, \bar{w})$ is not in the continuation region, then neither is $(x, y)$ for any $x \leq \underline{w}, y \geq \bar{w}$. This means that once $(\underline{w}, \bar{w})$ leaves the continuation region, no further crossing of $[\underline{w}, \bar{w}]$ will happen. So if we first leave 
the continuation region by an increase of $\bar{w}$, then $\underline{w}$ will not go any lower; we would always choose to stop before that happened. This would be the case of a successful fund which has risen in value; the manager will stop only if the fund level falls far enough from the maximum to endanger the gains. We find that actually only a small fall will trigger stopping. If $(\underline{w}, \bar{w})$ leaves the continuation region by $\underline{w}$ falling, we are seeing an unsuccessful fund which has made significant losses. In this case we see that the stopping barrier is actually quite high; the manager will keep on gambling in the hope of recovering some of the losses and will either gamble to extinction or until enough of the losses have been recovered that he will choose to stop.

\section{Conclusions}

We have taken the problem of a fund manager whose objective is to maximize the expected utility of his wealth, which is made up of a performance fee and a management fee. Under certain simplifying assumptions we argue that his objective is a function only of the terminal level of the fund, and the maximum and minimum levels achieved by the fund. A general argument equates the investment problem to a corresponding optimal stopping problem for Brownian motion, which we approximate by discretizing the Brownian motion to a random walk; in this form, the problem can be solved efficiently using numerical methods, and we illustrate the optimal stopping rule with some numerical examples.

While stopping problems for Brownian motion based on the value and the running maximum are much studied (see [1] for a seminal contribution), there has been less attention to stopping problems involving the value, the running maximum and the running minimum (though see the recent paper of Cox and Obloj [2] for an important contribution). The existing literature deals with such questions in the context of finding joint laws for the two (or three) variables $\bar{B}_{\tau}, B_{\tau}$ (and $\underline{B}_{\tau}$ ) which are extremal in some sense (see [6], where the stochastically largest maximum of a martingale whose terminal distribution is specified is shown to be achieved by the Azéma-Yor construction), and the analysis is typically quite detailed. The flavour of the present study is somewhat different however, and we readily turn to numerical methods because the problem is too complicated to be amenable to analysis.

\section{Appendix A. Proof of Lemma 2.1}

First we prove that

$$
\sup _{\theta} \mathbb{E} F\left(\underline{w}_{1}, w_{1}, \bar{w}_{1}\right) \geq \sup _{T \in \mathcal{T}} \mathbb{E} F\left(\underline{B}_{T}, B_{T}, \bar{B}_{T}\right) .
$$

If $T \in \mathcal{T}$, the process

$$
w_{t} \equiv B\left(\frac{t}{1-t} \wedge T\right)
$$

may be represented as

$$
w_{t}=\int_{0}^{t} \mathbf{1}\left\{s \leq T^{\prime}\right\} \frac{\mathrm{d} W_{s}}{1-s},
$$

where $T^{\prime}=T /(1+T)$ and $W$ is the standard Brownian motion defined by

$$
\int_{0}^{t} \frac{\mathrm{d} W_{s}}{1-s}=B\left(\frac{t}{1-t}\right) .
$$

Accordingly, $\left(\underline{w}_{1}, w_{1}, \bar{w}_{1}\right)=\left(\underline{B}_{T}, B_{T}, \bar{B}_{T}\right)$, proving the first inequality. 
For the converse inequality, we first note that

$$
\sup _{\theta} \mathbb{E} F\left(\underline{w}_{1}, w_{1}, \bar{w}_{1}\right)=\sup _{\theta \in S} \mathbb{E} F\left(\underline{w}_{1}, w_{1}, \bar{w}_{1}\right)=\sup _{\varepsilon} \sup _{\theta \in S_{\varepsilon}} \mathbb{E} F\left(\underline{w}_{1}, w_{1}, \bar{w}_{1}\right),
$$

where $\delta$ is the vector space of simple processes

$$
\theta=\sum_{j=0}^{n} Z_{j} \mathbf{1}_{\left\{t_{j}<t \leq t_{j+1}\right\}}
$$

for some $0=t_{0}<t_{1}<\cdots<t_{n+1}=1$, and $Z_{j} \in L^{\infty}\left(\mathcal{F}_{t_{j}}\right)$ for all $j$, and $\S_{\varepsilon}=\{\theta \in s:|\theta| \geq$ $\varepsilon\}$. So it will be sufficient to show that whenever we have some $\theta \in \delta_{\varepsilon}$ then there is some Brownian motion $B$ with canonical filtration $\left(\mathscr{B}_{t}\right)$ and a $\left(\mathscr{B}_{t}\right)$-stopping time $T$ such that

$$
\mathbb{E} F\left(\underline{w}_{1}, w_{1}, \bar{w}_{1}\right)=\mathbb{E} F\left(\underline{B}_{T}, B_{T}, \bar{B}_{T}\right) .
$$

Given $\theta \in \varsigma_{\varepsilon}$ of the form (A.1), we form the quadratic variation process

$$
A_{t}=\int_{0}^{t} \theta_{s}^{2} \mathrm{~d} s,
$$

and define $B_{t}=w\left(\tau_{t}\right)$, where $\tau$ is the continuous strictly-increasing inverse to $A$. Next define

$$
T_{k}=A_{t_{k}}=\sum_{j=0}^{k-1} Z_{j}^{2}\left(t_{j+1}-t_{j}\right) .
$$

We claim that for each $k, \mathcal{B}_{s}=\mathcal{F}_{\tau_{s}}$ for all $0 \leq s \leq T_{k}$. It is clear that $\mathscr{B}_{T_{k}} \subseteq \mathcal{F}_{t_{k}}$, but we shall prove by induction that equality holds for all $k$. Evidently equality holds for $k=0$, since both $\sigma$-fields are trivial. Suppose it is true up to some value of $k$. Then $\mathcal{B}\left(T_{k}\right)=\mathcal{F}\left(t_{k}\right)$, and so $Z_{k}$ is $\mathcal{B}\left(T_{k}\right)$-measurable. Now, for $0 \leq s \leq T_{k+1}-T_{k}$, we have

$$
B_{T_{k}+s}-B_{T_{k}}=Z_{k}\left\{w\left(t_{k}+s Z_{k}^{-2}\right)-w\left(t_{k}\right)\right\}
$$

and, therefore, we can deduce the path $\left(w\left(t_{k}+u\right)\right)_{0 \leq u \leq t_{k+1}-t_{k}}$ from the path of $\left.\left(B_{u}\right)_{0 \leq u \leq T_{k+1}}\right)$, since $Z_{k}$ is $\mathscr{B}\left(T_{k}\right)$-measurable. This extends the conclusion out to $k+1$ and, hence, for all $k$, and the equality (A.2) follows from taking $k=n+1$.

\section{Appendix B. Proof of Lemma 2.2}

For $h>0$ we are going to embed a scaled random walk in our Brownian motion. Let, therefore, $\sigma_{0}^{h}=0$ and

$$
\sigma_{n+1}^{h}=\inf \left\{t>\sigma_{n}^{h} ;\left|B_{t}-B_{\sigma_{n}^{h}}\right|=h\right\}, \quad n \geq 0 .
$$

Then $w_{n}^{h}=B_{\sigma_{n}^{h}}, n \geq 0$ clearly defines a random walk on $B_{0}+h \mathbb{Z}$ satisfying

$$
\left\|\left(\underline{B}_{t}, B_{t}, \bar{B}_{t}\right)-\left(\underline{w}_{n}^{h}, w_{n}^{h}, \bar{w}_{n}^{h}\right)\right\| \leq h \sqrt{3}
$$

for $\sigma_{n-1}^{h} \leq t \leq \sigma_{n+1}^{h}, n \geq 1$. Any $w^{h}$-stopping time $\tau$ naturally induces a $B$-stopping time $\hat{\tau}=\sigma_{\tau}^{h}$ with $w_{\tau}^{h}=B_{\hat{\tau}}$, giving

$$
V \geq V^{h}
$$


Moreover, for any $B$-stopping time $\tau$

$$
\tau^{h}=\inf \left\{n \geq 0 ; \sigma_{n}^{h} \geq \tau\right\}
$$

defines a random time with

$$
\left|w_{\tau^{h}}^{h}-B_{\tau}\right| \leq h, \quad\left|\bar{w}_{\tau^{h}}^{h}-\bar{B}_{\tau}\right| \leq h, \quad\left|\underline{w}_{\tau^{h}}^{h}-\underline{B}_{\tau}\right| \leq h .
$$

However, $\tau^{h}$ is not necessarily a $w^{h}$-stopping time. Define $g_{n}^{h}=\sigma\left(w_{j}^{h}: j \leq n\right)$ and $g^{h}=$ $\sigma\left(w_{j}^{h}: j \geq 0\right)$. Now let $\pi_{n}=P\left[\tau \in\left(\sigma_{n}^{h}, \sigma_{n-1}^{h}\right] \mid g^{h}\right]$. Note that

$$
\pi_{n}=P\left[\tau \in\left(\sigma_{n-1}^{h}, \sigma_{n}^{h}\right] \mid g_{n}^{h}\right]
$$

since $g_{n}^{h}=\sigma\left(\xi_{j}: j=1 \ldots n\right)$, with $\xi_{j}$ independent and identically distributed. Now we give ourselves a uniform random variable $U$, independent of $B$ and add this to every $g_{n}^{h}$ to form $\tilde{g}_{n}^{h}=g_{n}^{h} \vee \sigma(U)$. If we stop the random walk $w_{n}^{h}$ at the $\tilde{g}^{h}$-stopping time

$$
S=\inf \left\{n: \sum_{i=1}^{n} \pi_{i}>U\right\} .
$$

we find that $w_{S}^{h}$ has the same law as $w_{\tau^{h}}^{h}$ and then

$$
V^{h} \geq V-\psi(h \sqrt{3}) \text {. }
$$

Combining (B.1) and (B.2) proves the desired result.

\section{References}

[1] AzÉma, J. And Yor, M. (1979). Une solution simple au problème de Skorokhod. In Séminaire de Probabilités XIII, Springer, Berlin, pp. 90-115.

[2] Cox, A. M. G. ANd OBLoJ, J. (2011). Robust hedging of double touch barrier options. SIAM J. Financial Math. 2, 141-182.

[3] Dybvig, P. H. And Koo, H.-K. (1996). Investment with taxes. Working paper.

[4] Goetzmann, W. N., Ingersoll, J. E. And Ross, S. A. (2003). High-water marks and hedge fund management contracts. J. Finance 58, 1685-1718.

[5] Guasoni, P. and Oblou, J. (2013). The incentives of hedge fund fees and high-water marks. Math. Finance. Available at: doi: 10.1111/mafi.12057.

[6] Rogers, L. C. G. (1993). The joint law of the maximum and terminal value of a martingale. Prob. Theory Relat. Fields 95, 451-466. 